Biology | Gun-Sik Park

\section{Moisture regulation in finger pad ridges can ensure optimal grip}

The ridges on the pads of our fingers, and the fingerprints we produce, have several benefits, including identification

purposes. However, this was

of ridged skin on the fingos pad, and it is more likely to

have evolved to optimise grip and touch. Professor Gun-Sik Park, Seoul National University, has explored how the finger pad has its own moisture regulation mechanisms to
ensure optimal grip in both we ensure optimal grip in
and dry conditions.

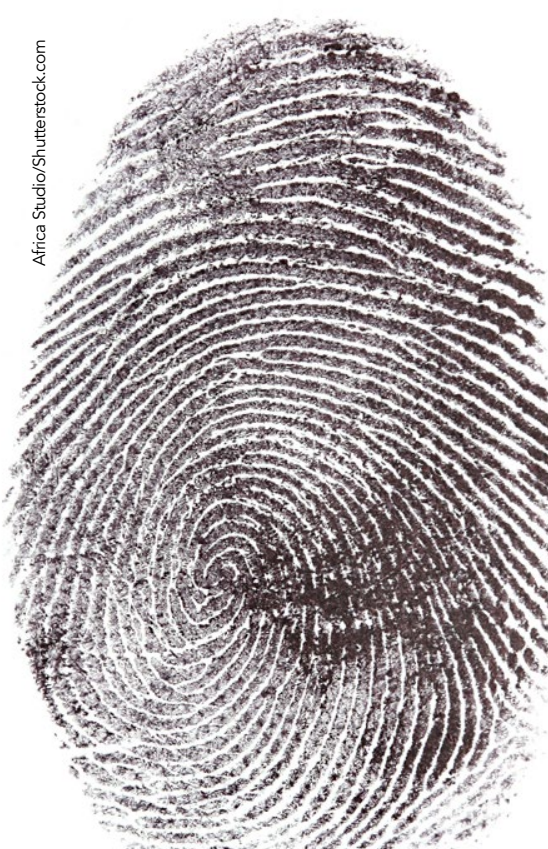
mechanisms by which ridges on the unclear. In mame hases, ridges have been shown to increase friction through interlocking contacts with a surface but may also reduce frictio moisture plays an important role, however too much moisture can lead to slipping. The hands and feet also have a much greater density of sweat glands than flat skin, althoug and stress rather than functioning

Professor Gun-Sik Park and colleagues at Seoul National University have explored this in further detail and undiscovered moisture regulation mechanism for optimising the grip of ridged skin.

MOISTURE REGULATION IN FINGER PADS The researchers used a variety of imaging techniques to explore the level of grip.

The results showed that regardless of whether the finger pad was wet or dry, there was a consistent volume of
moisture within the furrows. This is likely the level of moisture required for optimal grip. In addition, the researchers found that the level of friction seemed to be higher in the thumb, compared to the little finger. This would also correspond to an contact with an item.

Interestingly, there appears to be a midground for moisture; if the finger pad is too wet, moisture levels will decrease but for the dry finger $p$ moisture levels will increase.

Initially, after the water droplet is inse the finger pad and area. This water is then gradually disseminated through the furrows on the finger pad, instigating a drying phenomenon. The imag used by the researchers also showed that this evaporation rate varied across the finger pad, depending on the depth of the furrows and where they are located on the finger pad. Moisture also

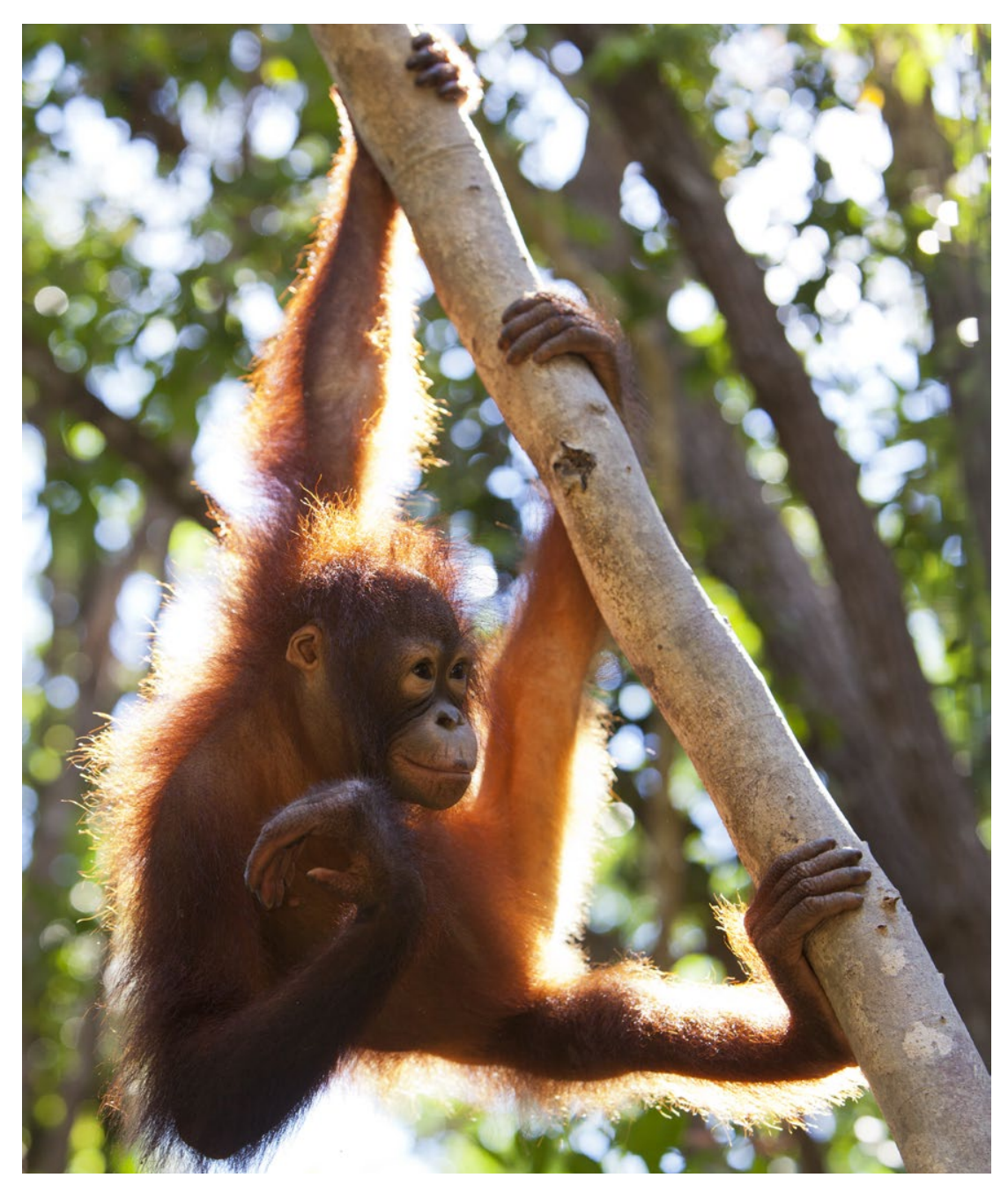
a surface It is a co thou area with seems to accumulate in the base of the furrow. The furrows towards the side of

the finger appear to siphon moisture to

evaporate. They do this by creating a low resistance pathway for the water move through

A fingerprint is an impression left on a surface, made by friction ridges on the finger.
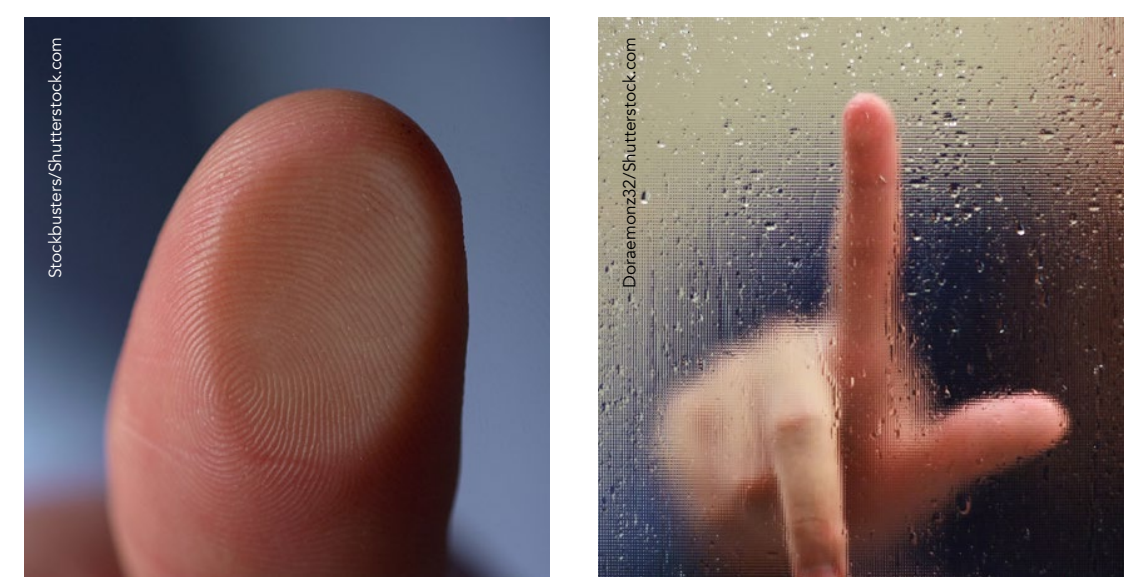

The study also showed that this was added to ridged skin, the same a fect was not seen with flat skin such as that on the chest, forearm, or thigh. the volume of moisture did not equalise as it did with the ridged finger pad. This may be explained in part by the increased levels of sweat secretion in tat skin compared to ridged skin.

may initially be thought that due to the ridged nature of the finger pad, Prex a sunface. However, Professor Park explains that Hother factors such as vel of plasticity must

MECHANISMS FOP

OPTIMISING GRIP

Using imaging techniques, the an optimal moisture level for flat skin, as evaporation only happened when liquid 

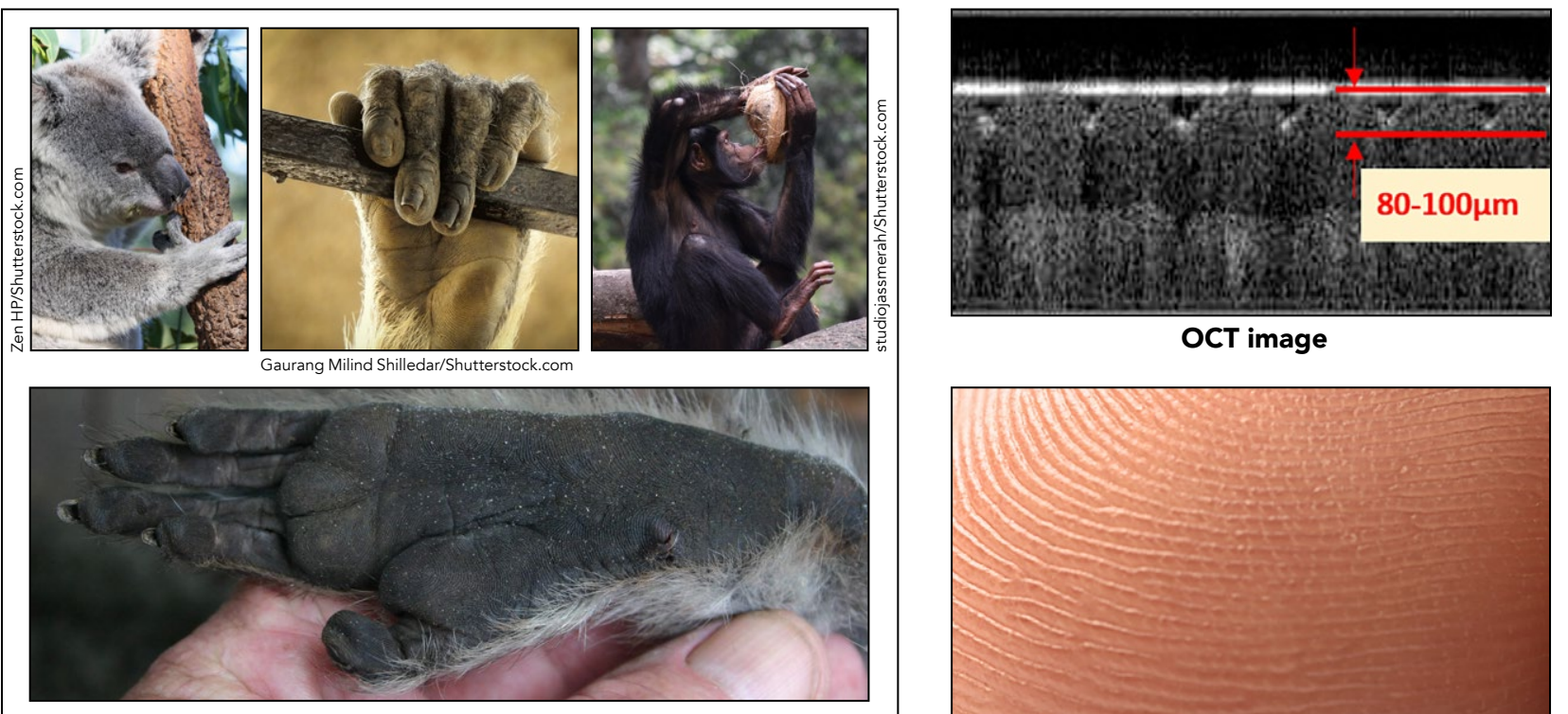

OCT image

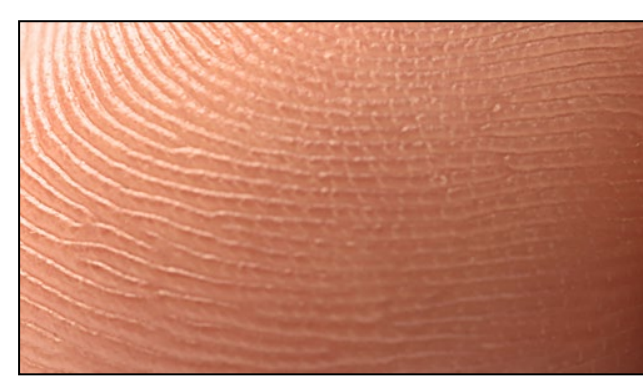

Fingerprints are unique to primates and koalas bu
pads of carnivorans, eg, feline or ursine species? moisture on a finger pad can reduce
friction and increase the risk of slipping. microchannels on the finger. This also ridges are able to maintain optimal moisture levels for grip.

The ability to regulate moisture levels through evaporation is key when we consider that excessive levels of

Overall, the results suggested that two different mechanisms are at play. The first allows ridges to act as a microfluidic array, moving fluid through promotes the evaporation of excess moisture whilst stil allowing some to

of sweat secretion and a steady state hydration level which is optimal for friction and grip.

Both mechanisms help to maintain the optimal amount of moisture in the furrows to maximise grip, regardless of whether a finger pad is initially wet or dry. Professor Park explains that these evolutionary adaptations have allowed primates to utilise manipulative and locomotive something which may have given then an advantage over other animals. A film of moisture between the ridges of the finger pad and the contact increase the risk of slipsing fromething that may be catastrophic for a primate Secondly, when moisture is present, the to the gripped sufface and subsequently block sweat pores.
If the ridges of a dry finger pad are pressed tightly to the surface, then sweat pores essentially become blocked. Whilst a wet finger pad will initially have unblocked sweat pores, the ridges become plasticised (more flexible and mouldable) over time. This contact between the finger pad and the result of this close contact is a cessation retention of moisture through capillary abilities in both wet and dry conditions, high in a tree. The interaction of ridges to the gripped surface and subsequently softening of the ridges allows a closer
Using imaging techniques, the researchers explored how finger pad ridges are able to maintain optimal moisture levels for grip. with a surface is complex and involves action, as well as a softening of the ridges which reduces the space where a film could form. Both of these event prevent a film
improve grip.

\section{MPROVING TECHNOLOGIES} required the use of slass as a contact surface due to its transparency for imaging. However, natural surfaces are rough and can be permeable to moisture. For example, materials such as paper sweat and decrease friction over time. The ability to soften and mould to a surface may be one way in which the ridges on finger pads overcome this challenge.

The work of the Seoul National University research team provides evidence of the underlaying mechanisms used by finger pads to regulate moisture, and the side of the finger pad funcows finger pad retain sufficient moisture to ensure the ridges are plasticised and conform to their conform to their
contact surface. Professor Park's research helps improve existing knowledge of the evolutionary benefit of fingerprints. In addition, a better understanding of the friction of finger pads has important connotations for progressing technologies, such as touch screens, and may help us understand the side the finger pad encourage

\section{Behind the Research}

\section{Professor Gun-Sik Park}

E: gunsik@snu.ac.kr T: $\quad$ +82-10-5408-7749 T: +82-31-888-9077 W: http://terahertz.snu.ac.kr

Research Objectives

Professor Gun-Sik Park and colleagues investigate the functionality of fingerprint ridges.

\section{Detail}

Bio

Gun-Sik Park received a PhD degree in physics from the University of in 1989. Since 1995, he has, USA in Professor at the Department of Physics Education and Department of Physics and Astronomy, Seoul National University and he became Professor Emeritus in 2021. Currently, he is Director of the Center for THz-driven Biological Systems in SNU which is sponsored by National Research Foundation and Ministry of Science and ICT of Korea. He has been an Honorary Professor of Samarkand State University in Uzbekistan since 2020
Funding This work was funded by the National Research Foundation Kover Telecommunication (MSIT) under Grants 2016R1A3B1908336.

\section{Collaborators}

- Seoung-Mok Yum, Seontae Kim Dongpyo Hong, Kyunghoon Jung, Juhan Kim, Kihoon Eom, Seonmyeong Kim, Jeongmin Jang, In-Keun Baeka, Matlabjon Kim, Michael J. Adams, and GunKim, Michael J. Adams, and Gun- Center for THz-Driven Biomedical Information Communicationce and

Systems, Department of Physics and Astronomy, College of Natural Sciences, Seoul Nation University, Seoul, Center for Advanced Institute of C Research, Advanced Institute of Convergen Sechnology, R\&D Department; Biology Research Institute, Department of Dermatology, College of Medicine, Yonsei University, Seoul; Department of Biomedical Engineering, College of Medicine, Seoul National University School of Chemical Engineering, University of Birmingham,

Birmingham GL51 9RE, United Kingdom; Institute of Applied Physics, Seoul National University.

\section{References}

Yum, SM Baek, IK Hong, D Kim, J Jung, K Kim, S Eom, Jang, J Kim, S Sa) Fin, M L regulate grip. Proceedings of the National $\mathrm{Academy}$ of Sciences of the United States of America, 117(50), 3166531673. https://doi.org/10.1073/pnas.2001055117

\section{Personal Response}

\section{Do you plan to do further research into why and} how fingerprint ridges help grip?

II The ways we will try to extend this research
include developing a deeper understanding of the physics behind this phenomena and observing 